\title{
Editorial
}

Nephrology

\section{Rivaroxaban in Chronic Hemodialysis Patients}

\author{
Heather Muster ${ }^{a}$ Harry Alcorn Jr. ${ }^{b}$ \\ ${ }^{a}$ Adjunct Faculty College of Pharmacy, University of Minnesota, and ${ }^{\mathrm{b}}$ DaVita Clinical Research, \\ Minneapolis, Minn., USA
}

The end-stage renal disease (ESRD) hemodialysis population in the United States at the end of 2013 was just over 421,000 people. The prevalence rate for chronic kidney disease (CKD) is approximately $14 \%$ in the general population with this CKD population dialysis, and CKD patients, often present with diagnoses that may make them candidates for anti-coagulation such as venous thromboembolic disease and atrial fibrillation $[1,2]$. Anticoagulation is not universally accepted in dialysis patients for some conditions such as atrial fibrillation. According to the 2014 American Heart Association guidelines, in those dialysis patients with atrial fibrillation and with a CHA2DS2-VASc score of $\geq 2$, it is reasonable to consider anti-coagulation with warfarin (listed as a class IIb recommendation) but this is controversial and not universally accepted. In fact, the Canadian Cardiovascular Society updated their recommendations in 2014 and recommended not routinely anti-coagulating non-valvular atrial fibrillation in patients with an $\mathrm{eGFR}<30 \mathrm{ml} / \mathrm{min}$ or on dialysis [3].

When anticoagulation is initiated, the usual agent selected is warfarin. While it is a well-studied and widely available medication, it presents challenges related to dietary requirements, monitoring and long-term compliance [4]. Finding alternative regimens would be welcome, but to date studies assessing the efficacy and safety of other oral anti-coagulants have not been routinely done in late stage kidney disease nor dialysis, which brings light

\section{KARGER}

E-Mail karger@karger.com

www.karger.com/ajn to this study using the novel oral anti-coagulant (NOAC), rivaroxaban.

In this issue, Dias et al. [1] report on the pharmacokinetics and the pharmacodynamics of rivaroxaban in a chronic dialysis population. Rivaroxaban is an anti-coagulant in the class of direct factor Xa inhibitors that has been approved for the prevention and treatment of deep vein thrombosis (DVT) and related diseases as well as prevention of stroke in patients with atrial fibrillation. It is not currently approved for the treatment of DVT in any patient with an eGFR of $<30 \mathrm{ml} / \mathrm{min}$ nor for stroke prevention in patients with an eGFR of $<15 \mathrm{ml} / \mathrm{min}$ in the United States. Canada guidelines do not advise its use in any patient with an eGFR of $<30 \mathrm{ml} / \mathrm{min}$.

Rivaroxaban has a dual elimination pathway. Approximately $66 \%$ of the drug is processed via the hepatic $\mathrm{P} 450$ system. Of those metabolites, half are eliminated fecally and half are excreted in the urine. The remaining unmetabolized drug (approximately one-third) is excreted in the urine as unchanged drug. It has been demonstrated that the renal elimination of unchanged drug is facilitated via active transport by the P-GP and Bcrp transporter proteins. Pharmacokinetic and pharmacodynamic studies in subjects with reduced renal function have proven that loss of renal function alters the clearance of rivaroxaban and increases the AUC [5].

Its molecular size is only $435 \mathrm{D}$ and it has a low volume of distribution of approximately 50 liters, both of which
(C) 2016 S. Karger AG, Basel

0250-8095/16/0434-0227\$39.50/0
Harry Alcorn Jr., PharmD

DaVita Clinical Research

825 S. 8th Street Minneapolis MN 55404 (USA)

E-Mail Harry.Alcorn@ davita.com 
render a molecule potentially dialyzable. However, it is also highly protein bound (>90\%) which limits the availability of drug to be removed even with high flux dialyzers. It could also be hypothesized that there may be alterations in bioavailability of drug or alterations in the alternate (hepatic) pathways that would change the clearance in a dialysis population.

The authors found changes in the PK and PD in ESRD patients were comparable to changes seen in prior investigations of moderate and severe renal subjects, also demonstrating the drug was not impacted by dialysis between the groups. In the renal subjects, there was little impact on Cmax; and administration of rivaroxaban administration pre vs. post $\mathrm{HD}$ had a minimal effect on the plasma AUC However the AUC as expected did increase $56 \%$ in hemodialysis patients. This closely correlated to the $64 \%$ increase in AUC noted by Kubitza et al. [5] in subjects with a $\mathrm{CrCl}$ of $<30 \mathrm{ml} / \mathrm{min}$. The magnitude of increased exposure is also consistent with the findings of De Vriese et al. [6] who studied a single $10 \mathrm{mg}$ dose in hemodialysis patients. In the De Vriese study, the AUC closely correlated to the results of Kubitza in both moderate and severe renal patients. However, De Vriese only found a half-life of $9 \mathrm{~h}$, which contrasts the current study of 12.2-13.2 h which one would expect in the renal and HD population [6].

During the study, no significant adverse events were reported except for 2 venous thromboses deemed unrelated to the rivaroxaban. As only a single dose was given, no conclusions can be drawn regarding the risk of accumulation. De Vriese et al. [6] did asses the AUC in hemodialysis patients after receiving a daily $10 \mathrm{mg}$ dose for 3 days. No significant accumulation or difference in AUC was noted compared to the single dose. This is reassuring but also not able to adequately predict the potential risk with chronic use.

The greatest risk with anticoagulation is bleeding and the ESRD population is of particular concern. In this study, only low dose heparin was allowed which likely mitigates the risk. However, this strategy may not mimic clinical practice where maintaining patency of the dialyzer is important. Special attention to bleeding risk in the CKD population is needed, as several studies have demonstrated a notably higher rate of bleeding, typically GI in origin compared with those without renal disease [7-9] Fortunately, when bleeding occurs on warfarin, the effects of can be reversed using vitamin $\mathrm{K}$ and/or plasma. This generally is not true for NOACs although factor concentrates have been suggested [10]. There are currently no approved reversal agents for rivaroxaban; however, a promising antidote is under development. A phase 3 study in healthy volunteers between the age of 50 and 75 of andexanet alpha for the reversal of rivaroxaban in healthy was recently completed. The anti-factor Xa activity of apixaban and rivaroxaban was reduced 94 and $92 \%$, respectively. Thrombin generation was fully restored in the apixaban-treated subjects and was $96 \%$ restored in rivaroxaban-treated subjects [11]. Should andexanet progress to approval, this would potentially offer patients with CKD a desirable option for anticoagulation.

CKD patients, in particular dialysis patients, are underrepresented in most clinical trials. This leaves clinicians with only their experience and extrapolated data to guide them in treatment decisions. Studies to evaluate the dosing strategies and outcomes in this population are critical for good patient care. This current study is an important first step to providing critical information on which to design longer treatment studies for novel anticoagulants.

\section{References}

1 Dias C, et al: Pharmacokinetics, pharmacodynamics, and safety of single-dose rivaroxaban in chronic hemodialysis. Am J Nephrol 2016, in press.

2 Daneschvar HL, Seddighzadeh A, Piazza G, Goldhaber SZ: Deep vein thrombosis in patients with chronic kidney disease. Thromb Haemost 2008;99:1035-1039.

3 Verma A, et al: 2014 focused update of the Canadian cardiovascular society guidelines for the management of atrial fibrillation. Can J Cardiol 2014;30:1114-1130.

4 Yao X, et al: Effect of adherence to oral anticoagulants on risk of stroke and major bleed- ing among patients with atrial fibrillation. J Am Heart Assoc 2016;5:pii:e003074.

5 Kubitza D, et al: Effects of renal impairment on the pharmacokinetics, pharmacodynamics and safety of rivaroxaban, an oral, direct factor Xa inhibitor. Br J Clin Pharmacol 2010; 70:703-712.

6 De Vriese AS, et al: Dose-finding study of rivaroxaban in hemodialysis patients. Am J Kidney Dis 2015;66:91-98.

7 Limdi NA, et al: Kidney function influences warfarin responsiveness and hemorrhagic complications. J Am Soc Nephrol 2009;20: 912-921.
8 Olesen JB, et al: Stroke and bleeding in atrial fibrillation with chronic kidney disease. $\mathrm{N}$ Engl J Med 2012;367:625-635.

9 Shah M, et al: Warfarin use and the risk for stroke and bleeding in patients with atrial fibrillation undergoing dialysis. Circulation 2014;129:1196-1203.

10 Siegal DM, Cuker A: Reversal of novel oral anticoagulants in patients with major bleeding. J Thromb Thrombolysis 2013;35:391398.

11 Siegal DM, et al: Andexanet alfa for the reversal of factor Xa inhibitor activity. N Engl J Med 2015;373:2413-2424. 